\title{
The role of inhibition by phosphocitrate and its analogue in chondrocyte differentiation and subchondral bone advance in Hartley guinea pigs
}

\author{
YUBO SUN, ALEX J. KIRALY, MICHAEL COX, DAVID R. MAUERHAN and EDWARD N. HANLEY JR.
}

Department of Orthopedic Surgery, Carolinas Medical Center, Charlotte, NC 28232, USA

Received July 27, 2017; Accepted November 10, 2017

DOI: $10.3892 / \mathrm{etm} .2018 .5846$

\begin{abstract}
Phosphocitrate (PC) and its analogue, PC- $\beta$ ethyl ester, inhibit articular cartilage degeneration in Hartley guinea pigs. However, the underlying molecular mechanisms remain unclear. The present study aimed to investigate the hypothesis that PC exerted its disease-modifying effect on osteoarthritis (OA), in part, by inhibiting a molecular program similar to that in the endochondral pathway of ossification. The results demonstrated that severe proteoglycan loss occurred in the superficial and middle zones, as well as in the calcified zone of articular cartilage in the Hartley guinea pigs. Subchondral bone advance was greater in the control Hartley guinea pigs compared with PC- or PC analogue-treated guinea pigs. Resorption of cartilage bars or islands and vascular invasion in the growth plate were also greater in the control guinea pigs compared with the PC- or PC analogue-treated guinea pigs. The levels of matrix metalloproteinase-13 and type $\mathrm{X}$ collagen within the articular cartilage and growth plate were significantly increased in the control guinea pigs compared with $\mathrm{PC}$-treated guinea pigs $(\mathrm{P}<0.05)$. These results indicated that articular chondrocytes in Hartley guinea pigs exhibited a hypertrophic phenotype and recapitulated a developmental molecular program similar to the endochondral pathway of ossification. Activation of this molecular program resulted in resorption of calcified articular cartilage and subchondral bone advance. This suggests that PC and PC analogues exerted their OA disease-modifying activity, in part, by inhibiting this molecular program.
\end{abstract}

Correspondence to: Dr Yubo Sun, Department of Orthopedic Surgery, Carolinas Medical Center, 1000 Blyth Boulevard, PO Box 32861, Charlotte, NC 28232, USA

E-mail: yubo.sun@carolinas.org

Abbreviations: COL-X, type X collagen; H\&E, hematoxylin and eosin; MMP13, matrix metalloproteinase-13; OA, osteoarthritis; PC, phosphocitrate; $\mathrm{PC}-\mathrm{E}$, phosphocitrate- $\beta$ ethyl ester

Key words: osteoarthritis, cartilage, subchondral bone, growth plate, calcification, phosphocitrate, type $\mathrm{X}$ collagen, matrix metalloproteinase-13

\section{Introduction}

Osteoarthritis (OA) is a heterogeneous and multifactorial degenerative joint disease characterized by joint pain, cartilage degradation and osteophyte formation. Approximately $86 \%$ of the societal costs of arthritis are estimated to be attributable to OA (1) however, there are currently no therapeutic agents available that halt the progression of articular cartilage degeneration. Non-surgical treatments for OA, including non-steroid anti-inflammatory drugs, oral steroid or knee steroid injection provide temporary symptomatic relief only (2). Therefore, the development of a structural OA disease-modifying drug that provides symptomatic relief and arrests the progression of cartilage degeneration is required. The lack of progress in the development of structural OA disease-modifying drugs is primarily due to limited understanding of the pathogenesis of OA and insufficient knowledge regarding the molecular targets for therapeutic intervention.

The biochemical events associated with the initiation or progression of OA are poorly understood. Extracellular matrix-degrading enzymes and inflammatory cytokines, including matrix metollproteinase-13 (MMP-13), ADAM metallopeptidase with thrombospondin type 1 motif 5 , interleukin-1 and cyclooxygenase-2 have been implicated in OA (3-5). The activation and abnormal phenotypic change of articular chondrocytes have also been implicated. Chondrocytes in OA articular cartilage exhibit an aberrant phenotype (6-8), although the exact mechanisms of chondrocyte activation and differentiation in OA articular cartilage remain unclear. Age-associated changes in extracellular matrix molecules or expression of inflammatory cytokines and growth factors may serve a role in the activation and differentiation of articular chondrocytes (7-9). Alternatively, articular chondrocytes may obtain properties with similarities to those of terminal differentiating chondrocytes in the growth plate. Terminal differentiating OA articular chondrocytes are associated with calcification, apoptosis, neovascularization and ossification (7-11) and may participate in articular cartilage degeneration by secreting large amounts of MMP-13 (12-14).

The number of tidemarks, the lines bordering the calcified cartilage and non-calcified cartilage, increases with age in human articular cartilage (14). The movement of tidemarks toward the articular surface is expected to result in an increase in the thickness of calcified zone cartilage, however, 
the calcified zone cartilage instead becomes thinner during aging (14). This phenomenon indicates replacement of the calcified cartilage by subchondral bone in the aging process. $\mathrm{OA}$ is also associated with articular cartilage thinning $(15,16)$. This association suggests that the replacement of calcified cartilage by subchondral bone may serve a role in articular cartilage thinning or cartilage degeneration in OA (17).

Phosphocitrate (PC) is a powerful calcification inhibitor (18), which prevents soft tissue calcification (19). PC also inhibits calcium crystal-induced cell membrane damages, mitogenesis, expression of extracellular matrix-degrading enzymes and crystal-induced cell death (20-23). Based on these results, a hypothesis that PC is a potentially disease-modifying drug for calcium crystal-associated primary OA was previously postulated (24). A subsequent study demonstrated that PC inhibited meniscal calcification in the Hartley guinea pigs and the reduction in meniscal calcification was accompanied with decreased articular cartilage degeneration (25).

It was previously demonstrated that, in cell cultures, PC inhibited the expression of numerous genes implicated in $\mathrm{OA}$ in the absence of calcium crystals (26-28). These previous results indicate that $\mathrm{PC}$ is a disease modifying drug for calcium crystal-associated primary OA and injury-induced secondary posttraumatic OA therapy. However, the molecular mechanisms underlying the OA disease-modifying activity of $\mathrm{PC}$ remain unclear. The present study aimed to investigate the hypothesis that PC exerts its OA disease-modifying effect, in part, by inhibiting a molecular program similar to the endochondral pathway of ossification.

\section{Materials and methods}

Reagents. Safranin O-fast green and hematoxylin and eosin (H\&E) were obtained from Polysciences, Inc. (Warrington, PA, USA). Antibodies specific to MMP-13 (cat. no. LS-B3168) and type X collagen (COL-X; cat. no. ab49945) were obtained from LifeSpan BioSciences, Inc. (Seattle, WA, USA) and Abcam (Cambridge, MA, USA), respectively. Antibodies specific to runt-related transcription factor 2 (cat. no. bs-1134R) and sex determining region Y-box 9 (cat. no. TA335171) were obtained from Bioss Antibodies, Inc. (Woburn, MA, USA) and OriGene Technologies, Inc. (Rockville, MD, USA), respectively. Mouse immunoglobulin G was obtained from BioGenex (Fremont, CA, USA; cat. no. HK119-7M). Secondary reagent (ImmPRESS reagent kit) was obtained from Vector Laboratories, Inc. (Burlingame, CA, USA; cat. no. MP-7401-50 ml). PC and PC- $\beta$ ethyl ester (PC-E) were synthesized according to previously detailed procedures (29). All other reagents, including normal horse serum, bovine serum albumin and 3,3'-diaminobenzidine, were obtained from Sigma-Aldrich (Merck KgaA, Darmstadt, Germany).

Animals. Hartley guinea pigs develop OA spontaneously 3 months following birth and reach skeletal maturity at the age of $\sim 6$ months (30-32). The progressive degenerative changes in the articular cartilage closely resemble the degenerative changes in human OA articular cartilage; therefore, Hartley guinea pigs have been widely used to study the pathogenesis of OA and test disease modifying drugs (33-37). Treatment of Hartley guinea pigs with PC and PC-E has been previously described (38). Briefly, young male Hartley guinea pigs (mean body weight, 280 g; Charles River Laboratories, Inc., Wilmington, MA, USA) were housed individually in solid bottom cages and maintained in a temperature-controlled room $\left(18-20^{\circ} \mathrm{C}\right)$ that was regulated on a 12 -h light-dark cycle. Relative humidity of the room was maintained between 45-55\%. Guinea Pig Chow (cat. no. 5025; Ralston Purina Co., Chicago, IL, USA) and water were available ad libitum. Following acclimation, 15 guinea pigs (age, 5 weeks) were randomly divided into three groups. The first group $(n=5)$ received intraperitoneal injection of PC (40 $\mathrm{mg} / \mathrm{kg})$ twice per week for a period of 2 months, the second group $(n=5)$ received intraperitoneal injection of PC-E (40 mg/ $\mathrm{kg}$ ) twice per week for a period of 2 months, and the last group $(n=5)$ received intraperitoneal injection of physiological saline twice per week for 2 months. Partial medial meniscectomy was then performed on the right knee of all guinea pigs. At 1 week following surgery, administration of treatment (twice per week at the same dose) was resumed in each group and continued for a further 5 months. Guinea pigs were then euthanized. Hind limbs were collected, fixed in $10 \%$ formalin for $24 \mathrm{~h}$ at room temperature and transferred to $70 \%$ ethanol prior to further use. The present study was performed according to the guidelines set forth by the Institutional Animal Care \& Use Committee of Carolinas Medical Center (Charlotte, NC, USA), which also approved the animal protocol.

Histological examinations. The tibia plateaus of partial meniscectomied right knees were decalcified in a formic acid/sodium citrate solution (Thermo Fisher Scientific, Inc., Waltham, MA, USA) and cut in the coronal plane to produce two equal parts. The posterior part was embedded in paraffin and sectioned with a Leica RM2025 microtome (Leica Microsystems $\mathrm{GmbH}$, Wetzlar, Germany) to obtain $4-\mu \mathrm{m}$-thick sections. A total of 3 non-consecutive sets of sections obtained at $400-\beta \mathrm{m}$ intervals from each tibia plateau ( 9 total sections covering the central most degenerated area of the medial tibial plateau) were stained with Safranin-O for 5 min and countered stained with fast green for $5 \mathrm{~min}$ at room temperature. A total of 2 sections from each tibial plateau were also stained with $\mathrm{H} \& \mathrm{E}$ for $10 \mathrm{~min}$ at room temperature. Safranin-O-fast green-stained sections were graded according to criteria described previously (39) with modifications, as detailed in Table I.

Growth plate thickness measurement. Growth plate thickness was determined using Image J photo analysis software (version 1.8.0_45; National Institutes of Health, Bethesda, MD, USA). Briefly, images of Safranin-O-fast green-stained sections (3 sections from each tibial plateau) were captured at x4 magnification with a digital camera (Sony DXC-S500; Sony Corporation, Tokyo, Japan) equipped with a light microscope (Nikon Optiphot-2; Nikon Corporation, Tokyo, Japan). The growth plate and growth plate cartilage bars or islands (red colored) embedded within the subchondral bone were selected using the following image thresholding and LAB color space parameters (L:0/255, A: 145/255,B: 0/255). Following selection, the areas of growth plate (particle size setting, $\geq 0.1 \mathrm{~mm}^{2}$ ) and growth plate cartilage bars (particle size setting, $\geq 0.0001 \mathrm{~mm}^{2}$ and $<0.1 \mathrm{~mm}^{2}$ ) were measured. Growth plate thickness was calculated by dividing the measured area of growth plate with 
Table I. Semi-quantitative histological grading scheme for medial tibia plateau of the guinea pig.

\begin{tabular}{|c|c|c|}
\hline Parameter & Grade & Description \\
\hline \multirow[t]{9}{*}{ Cartilage structure } & 0 & Normal, smooth, uninterrupted surface \\
\hline & 1 & Mild surface irregularities, no clefts \\
\hline & 2 & Irregular surface, 1-3 superficial clefts \\
\hline & 3 & $>3$ clefts and/or loss of cartilage to superficial zone \\
\hline & 4 & 1-3 clefts extending into the middle zone \\
\hline & 5 & $>3$ clefts and/or loss of cartilage extending into the middle zone \\
\hline & 6 & $1-3$ clefts extending into the deep zone \\
\hline & 7 & $>3$ clefts extending into the deep zone and/or loss (5-20\%) of cartilage to deep zone \\
\hline & 8 & Fissures or loss of cartilage extended to the zone of calcified cartilage \\
\hline \multirow[t]{7}{*}{ Safranin-O staining } & 0 & Uniform staining throughout articular cartilage \\
\hline & 1 & Loss of staining in superficial zone only and for less than half the length of the plateau \\
\hline & 2 & Loss of staining in superficial zone for half the length or greater of the plateau \\
\hline & 3 & Loss of staining in superficial and middle zones for less than half the length of the plateau \\
\hline & 4 & Loss of staining in superficial and middle zones for half the length or greater \\
\hline & 5 & Loss of staining in all three zones for less than half the length of the condyle or plateau \\
\hline & 6 & Loss of staining in all three zones for half the length or greater of the plateau \\
\hline \multirow[t]{4}{*}{ Cellularity } & 0 & Uniform dense chondrocytes in the superficial zone \\
\hline & 1 & Cell density is reduced in the superficial zone \\
\hline & 2 & Cell density is reduced in both superficial zone and middle zone \\
\hline & 3 & Cell density is also reduced in deep zone \\
\hline \multirow[t]{3}{*}{ Tidemark } & 0 & Faint tidemark \\
\hline & 1 & Moderate visible tidemark \\
\hline & 2 & Strong visible tidemark \\
\hline \multirow[t]{5}{*}{ Subchondral bone advance } & 0 & Distance between subchondral bone front and tidemark is large (away more than 4 cells) \\
\hline & 1 & Distance between subchondral bone front and tidemark is moderate (roughly 3-4 cells) \\
\hline & 2 & Distance between subchondral bone front and tidemark is small ( 2 cells) \\
\hline & 3 & Distance between subchondral bone front and tidemark is extremely small ( 1 cell) \\
\hline & 4 & Subchondral bone front reaches to tidemark. \\
\hline
\end{tabular}

the length of the growth plate or by dividing the measured area of growth plate + the measured area of growth plate bars by the length of the growth plate. Normalized growth plate thickness was used in all analyses, with the growth plate thickness in the untreated guinea pigs set as 1 .

Immunostaining. A total of 2 sections from each tibial plateau were deparaffinized with xylene and rehydrated with graded ethanol. Endogenous peroxidase activity was blocked via incubation of these sections with deionized water containing $3 \%$ $\mathrm{H}_{2} \mathrm{O}_{2}$ for $15 \mathrm{~min}$ at room temperature. Non-specific binding was blocked via incubation with 1001 of $10 \%$ normal horse serum diluted in base solution (4\% bovine serum albumin and $5 \%$ non-fat dry milk in phosphate buffered saline) for $20 \mathrm{~min}$ at room temperature. Sections were subsequently incubated with primary antibodies (1:100) for $1 \mathrm{~h}$ at room temperature, followed with secondary reagent $(1: 200)$ for $30 \mathrm{~min}$ at room temperature. A negative control was performed using mouse immunoglubulin G. Slides were rinsed with PBS three times, visualized with 3,3'-diaminobenzidine for $5 \mathrm{~min}$ at room temperature and counterstained with light green for $5 \mathrm{~min}$ at room temperature. Slides were viewed under light microscopy and graded on a scale of $0-4$ as described previously $(38,40)$.
Briefly, 0, very weak staining; 1 , weak staining; 2 , moderate staining; 3 , strong staining; and 4, very strong staining.

Statistical analysis. All data, including histological scores of articular cartilages, growth plate thickness, articular cartilage thickness, total tissue volume, immunostaining scores of COL-X and MMP13 and the number of marrow spaces, were presented as the mean \pm standard deviation. The differences between groups were analyzed using one-way analysis of variance followed by a Tukey or Dunnett's test. Statistical analysis was performed using the statistical analysis tool in Sigma Plot software (version 12; Systat Software, Inc., San Jose, CA, USA). $\mathrm{P}<0.05$ was considered to indicate a statistically significant difference.

\section{Results}

$P C$ and $P C-E$ reduce the histological score of articular cartilage. Representative Safranin-O-fast green-stained sections are presented in Fig. 1A. Sections were previously examined and it was demonstrated that PC and PC-E inhibited articular cartilage degeneration in the guinea pig model of posttraumatic OA (38). Further examination of these sections 
in the present study indicated further differences between the articular cartilage in the untreated and PC-treated guinea pigs. The tidemark in untreated guinea pigs was more visible and closer to the articular surface compared with the tidemark in PC-treated guinea pigs. In addition, severe proteoglycan loss occurred in the superficial and middle zones, as well as in the calcified zone in the untreated guinea pigs, but not in the PC-treated guinea pigs. There were also fewer articular cartilage bars or islands embedded within the subchondral bone in the untreated guinea pigs compared with PC-treated guinea pigs. These differences indicated that the advance of calcification front and active replacement of the calcified cartilage by subchondral bone occurred in the Hartley guinea pigs and that PC inhibited this process. Therefore, subchondral bone advance toward articular cartilage surface or the tidemark may serve a role in articular cartilage thinning or destruction. Similar differences were also observed between the articular cartilage in untreated and PC-E-treated guinea pigs (data not shown).

Representative H\&E stained sections are presented in Fig. 1B. The subchondral bone was stained dark pink and articular cartilage was stained pink or light pink. Similar to Safranin-O-fast green staining, H\&E staining demonstrated that the tidemark in the untreated guinea pigs was more visible and closer to articular cartilage surface compared with the tidemark in PC-treated guinea pigs. The superficial, middle, deep and calcified zones of articular cartilage in PC-treated guinea pigs were stained light pink. By contrast, only the middle and deep zones in the untreated guinea pigs were stained light pink, whereas the superficial zone and the entire calcified zone were stained pink. These differences indicated that the extracellular matrices within the superficial and calcified zones in the untreated guinea pigs differed from those in PC-treated guinea pigs, suggesting that articular cartilage degeneration or matrix loss occurred in the superficial and calcified zones in the untreated guinea pigs. Chondrocyte clusters were observed in the articular cartilage of untreated guinea pigs, but rarely in the articular cartilage of PC-treated guinea pigs. In addition, there were fewer chondrocytes within the articular cartilage in the untreated guinea pigs compared with PC-treated guinea pigs. These results are consistent with previous studies that have demonstrated that chondrocytes were activated and chondrocyte apoptosis was prominent in OA articular cartilage (6,41-43).

All safranin-O-fast green-stained sections were graded. PC treatment significantly reduced the histological score of the medial tibia plateau cartilage, resulting in a $46 \%$ reduction in the histological score, graded according to the criteria described previously (39) and a 50\% reduction in the histological score, graded according to the criteria described in Table I, compared with the untreated control (Fig. 2A). PC-E treatment also reduced the histological scores, graded according to the criteria described previously (39) and the criteria described in Table I, significantly compared with the untreated control, however, the reductions were less marked compared with PC in the inhibition of articular cartilage degeneration (Fig. 2A). Select sub-category histological scores in the untreated, PC- and PC-E-treated guinea pigs are presented in Fig. 2B. $\mathrm{PC}$ treatment resulted in a 37,82 and $65 \%$ significant reduction in cellularity, tidemark and subchondral bone advance sub-scores, respectively. By contrast, PC-E treatment only resulted in a significant reduction in tidemark and subchondral sub-scores compared with the untreated control, whereas the difference in the sub-histological score for cellularity between the control and PC-E treated groups was not significant, which indicated reduced disease-modifying activity compared with PC treatment.

$P C$ and $P C-E$ inhibit growth plate thinning. In addition to cartilage degeneration, $\mathrm{PC}$ also inhibited growth plate thinning. The growth plate in PC-treated guinea pigs was markedly thicker than the growth plate in untreated guinea pigs (Fig. 3A). In addition, there were more growth plate cartilage bars or islands embedded within the metaphyseal bone in PC-treated guinea pigs compared with untreated guinea pigs. A similar phenomenon was observed in PC-E-treated guinea pigs (data not shown). The differences were quantified by measuring growth plate thickness using ImageJ software. The growth plates in PC- and PC-E-treated guinea pigs were 54 and $36 \%$ thicker compared with the growth plate in untreated guinea pigs, respectively (Fig. 3B). If the area of growth plate cartilage bars was also included in the calculation the growth plate in the PC- and PC-E-treated guinea pigs was 97 and $41 \%$ thicker compared with the growth plate in untreated guinea pigs, respectively.

PC and PC-E appeared to inhibit the conversion of growth plate cartilage into bone, therefore, the effects of treatment on metaphyseal bone was investigated by measuring the areas of bone matrices under the growth plate using ImageJ software. The differences between the areas of bone matrices, an indirect indicator of bone volume, in the untreated and $\mathrm{PC}$ or PC-E-treated guinea pigs were not significant (Fig. 3C). However, the total areas of matrices (areas of bone matrices + areas of growth plate cartilage bars or islands) in the PC-or PC-E-treated guinea pigs was significantly greater than that in the untreated guinea pigs (Fig. 3C).

Analyses using ImageJ software were also performed to determine the thickness of articular cartilage. The articular cartilage in the central most degenerated area of the medial tibial plateau cartilage in the PC- and PC-E-treated guinea pigs was 40 and $29 \%$ thicker compared with that in untreated guinea pigs, respectively and the differences were significant (Fig. 3D). If the area of articular cartilage bars or islands was also included in the calculation the articular cartilage in the central most degenerated area of the medial tibial plateau cartilage in the PC- and PC-E-treated guinea pigs was 53 and $38 \%$ thicker compared with the articular cartilage in the untreated guinea pigs, respectively.

$P C$ and PC-E inhibit vascular invasion into growth plate. Representative H\&E-stained sections of growth plates are presented in Fig. 4. The growth plate in the untreated guinea pigs was markedly thinner and had fewer growth plate cartilage bars compared with that in the PC or PC-E treated guinea pigs (Fig. 4A). In addition, there was an increased number of marrow spaces or vascular channels at the junction between the growth plate and metaphysis in the untreated guinea pigs compared with PC- or PC-E-treated guinea pigs. The number of marrow spaces is an indirect indicator of vascular invasion into the growth plate. The PC or PC-E treatment resulted in 

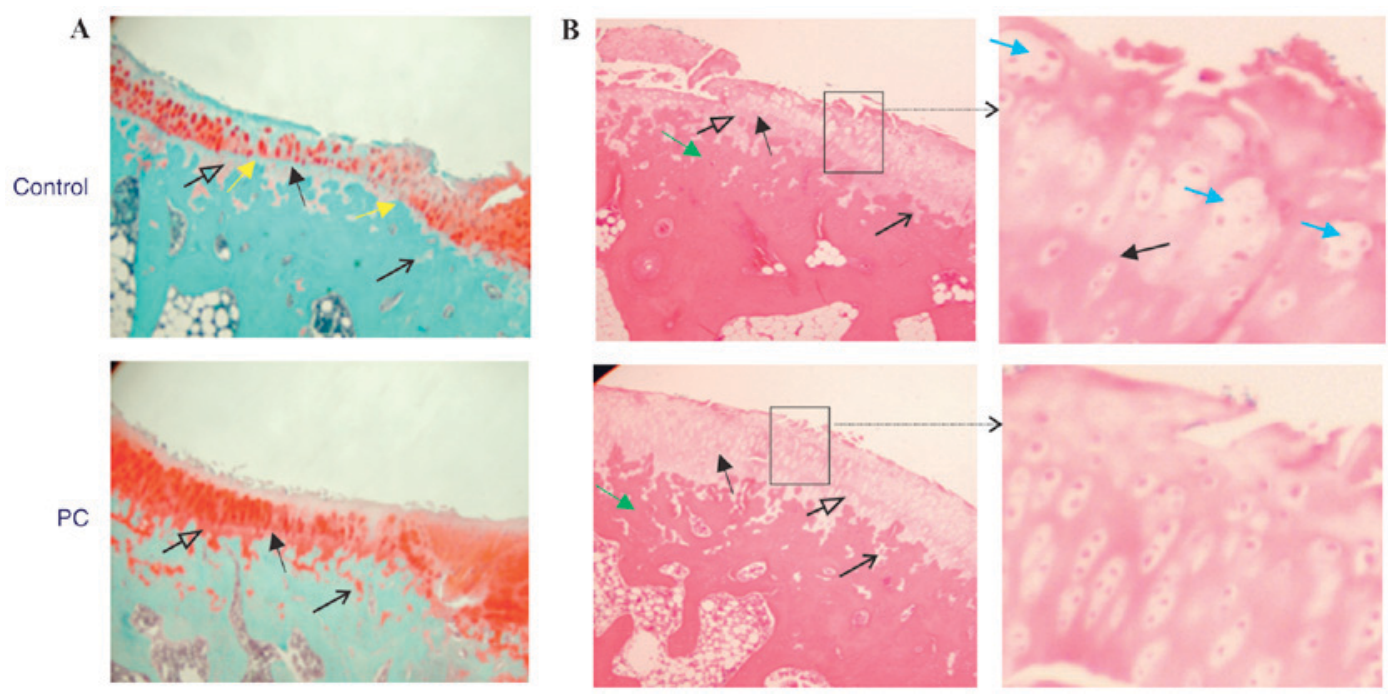

Figure 1. Histological stained-sections of articular cartilage. (A) Representative Safranin-O-fast green stained-sections of the central most degenerated area of medial tibial plateau cartilage (magnification, $\mathrm{x} 4$ ). Solid black arrows indicate the tidemark. Open black arrows indicate the calcified zone articular cartilage. Line black arrows indicate articular cartilage bars or islands embedded within subchondral bone. Yellow arrows indicate locations where subchondral bone has reached the tidemark. (B) Representative hematoxylin and eosin stained-sections of the central most degenerated area of medial tibial plateau cartilage (magnification, x4). Solid black arrows indicate the tidemark. Open black arrows indicate the calcified zone articular cartilage. Line black arrows indicate the articular cartilage bars or islands embedded within subchondral bone. Green arrows indicate the subchondral bone. Areas enclosed by a box are enlarged. Blue arrows indicate articular chondrocyte cluster (magnification, x10). PC, phosphocitrate.

reduced vascular invasion into the growth plate compared with the untreated group (Fig. 4B). There were 39 and $18 \%$ fewer marrow spaces or vascular channels at the junction in the PC- and PC-E treated guinea pigs compared with the untreated guinea pigs, respectively and these differences were significant.

$P C$ and $P C-E$ reduce the levels of COL-X and MMP-13 protein. Representative immunostaining images of the expression of COL-X are presented in Fig. 5A. High levels of COL-X were detected in the nucleus and cytoplasm of articular chondrocytes in the untreated guinea pigs. These positively stained chondrocytes were primarily located in the deep and calcified zones. Moderate or low levels of COL-X were detected in the extracellular matrices within the superficial and middle zones in the untreated guinea pigs. COL-X was also detected in the nucleus and cytoplasm of chondrocytes in the growth plate cartilage. The positively stained growth plate chondrocytes were primarily located in the maturation and hypertrophic zones. COL-X was also detected in the cells located at the junction between the growth plate and metaphyseal bone in the untreated guinea pigs. PC treatment reduced the levels of COL-X in the articular chondrocytes located in the deep and calcified zones, however, it had little effect on the levels of COL-X in the extracellular matrices within the superficial and middle zones. PC treatment reduced the levels of COL-X in the growth plate chondrocytes located in the maturation zone, hypertrophic zone and at the junction between growth plate and metaphyseal bone, as well as the levels of COL-X in the extracellular matrices within the middle zone compared with untreated samples. PC-E displayed minor inhibitory effects on the levels of COL-X.

Representative immunostaining images of the expression of MMP-13 protein are presented in Fig. 5B. MMP-13 protein was detected in the nucleus and cytoplasm of articular chondrocytes and pericellular and extracellular areas in the untreated guinea pigs. The highest levels of MMP-13 protein were detected in the superficial, middle and deep zones. By contrast, the highest levels of MMP-13 protein in the growth plate were detected in the maturation and hypertrophic zones. Consistent with its OA disease-modifying activities, PC treatment reduced the levels of MMP-13 protein in the articular cartilage and growth plate compared with the untreated group. In addition, consistent with its reduced inhibitory effects on cartilage degeneration, PC-E exhibited reduced inhibitory effects on the levels of MMP-13 protein compared with $\mathrm{PC}$. The levels of runt-related transcription factor 2 and sex determining region Y-box 9 were also investigated, however, the differences in the levels of these proteins between the untreated and PC-treated guinea pigs were not significant (data not shown).

Immunostaining scores of COL-X and MMP13 proteins are presented in Fig. 6. The immunostaining scores of COL-X in the articular chondrocytes and cells located at the junction between the growth plate and metaphyseal bone in the PC-treated guinea pigs were decreased by 32 and $37 \%$ compared with the scores in untreated guinea pigs, respectively and the differences were significant. The immunostaining scores of COL-X in PC-E-treated guinea pigs were also decreased compared with the score of COL-X in untreated guinea pigs, however, the differences were not significant. Immunostaining scores of MMP-13 protein in the articular chondrocytes and cells located within the hypertrophic zone of growth plate in the PC-treated guinea pigs were decreased by 49 and $37 \%$ compared with the score in the untreated guinea pigs, respectively and the differences were significant (Fig. 6B). The immunostaining scores of MMP-13 protein in PC-E-treated guinea pigs were also decreased compared with that in untreated guinea pigs, however, only the difference between the immunostaining 
A

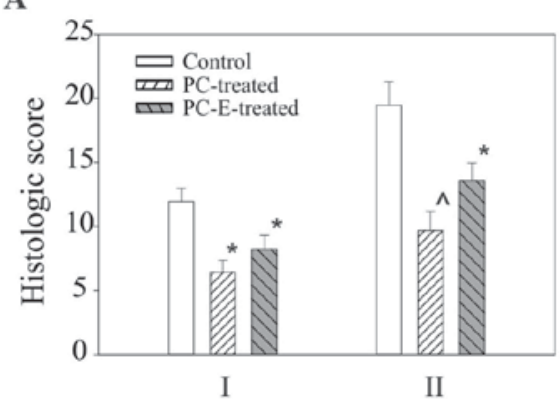

B

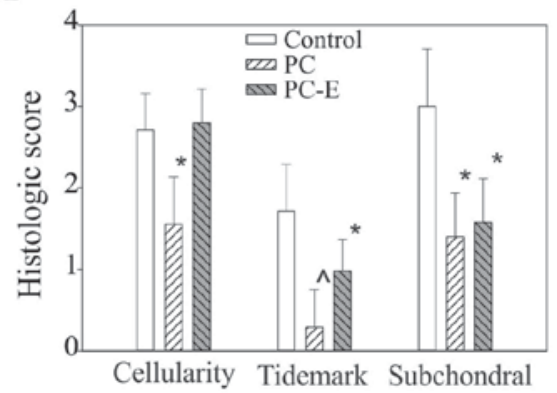

Figure 2. Histological scores of medial tibial plateaus cartilage. (A) I, Histologic scores, graded according to the criteria described previously (39), in untreated, PC-, and PC-E-treated guinea pigs were $11.95 \pm 1.05,6.43 \pm 0.93$ and $8.24 \pm 1.07$, respectively. II, Histologic scores, graded according to the criteria listed in

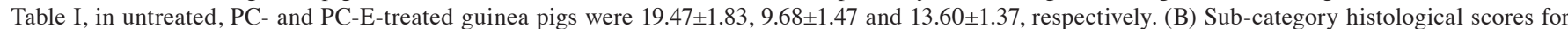
cellularity, tidemark and subchondral bone advance in untreated, $\mathrm{PC}$-, and $\mathrm{PC}-\mathrm{E}-$ treated guinea pigs. ${ }^{\mathrm{P}} \mathrm{P}<0.05$ vs. control; ${ }^{\wedge} \mathrm{P}<0.05$ vs. control and $\mathrm{PC}-\mathrm{E}$. PC, phosphocitrate; PC-E, PC- $\beta$ ethyl ester.

A

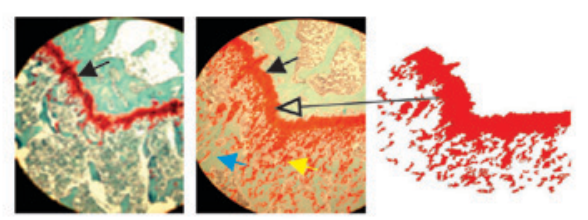

Untreated PC-treated ImageJ selected area
B

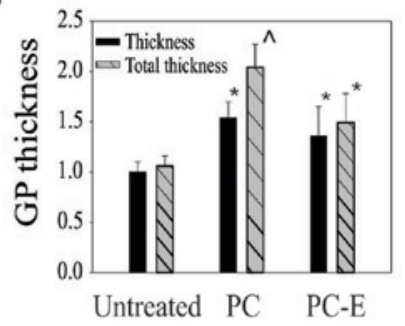

D

$\mathrm{C}$

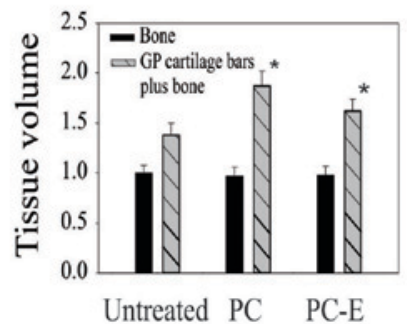

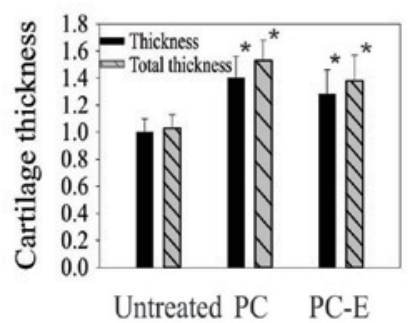

Figure 3. Thickness of GP and articular cartilages. (A) Representative Safranin-O-fast green stained-sections (magnification, $\mathrm{x} 4$ ). Solid black arrows indicate the GP. The yellow arrow indicates GP cartilage bars or islands. The blue arrow indicates metaphyseal bone. The long black arrow indicates GP and GP cartilage bars or islands. (B) GP thickness. Black bars indicate normalized or relative GP thicknesses. Grey bars indicate normalized total or relative total GP thicknesses. (C) Areas of metaphyseal bone matrices and total metaphyseal matrix (areas of metaphyseal bone matrices + areas of GP cartilage bars). (D) Thickness of the central most degenerated area of articular cartilage. ${ }^{*} \mathrm{P}<0.05$ vs. untreated; $\wedge \mathrm{P}<0.05$ vs. untreated and $\mathrm{PC}-\mathrm{E}$. PC, phosphocitrate; $\mathrm{PC}-\mathrm{E}$, PC- $\beta$ ethyl ester; GP, growth plate.

A

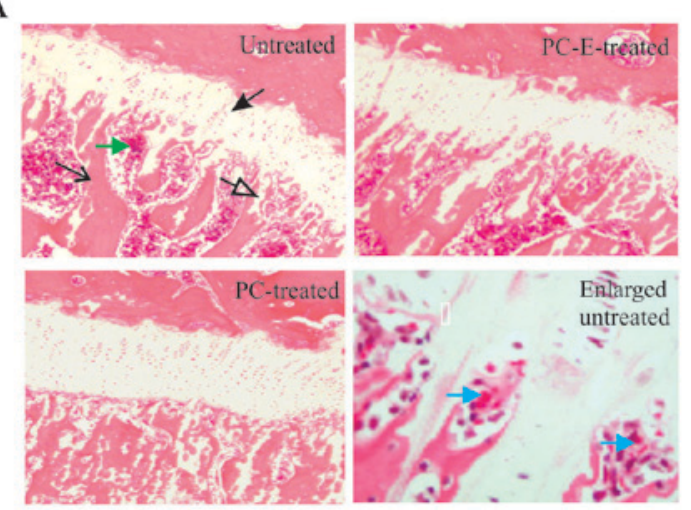

B

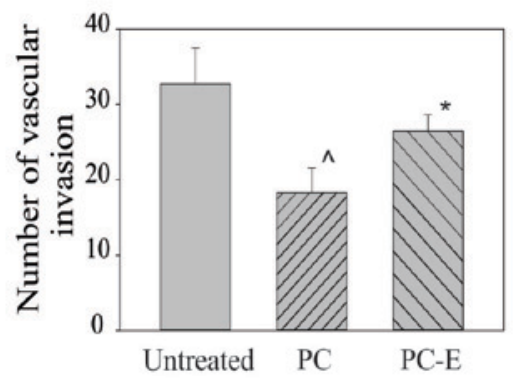

Figure 4. Effect of PC and PC-E on the growth plate and marrow space at the junction between the growth plate and metaphysis. (A) Representative hematoxylin and eosin stained-sections (magnification, $\mathrm{x} 4$ ). The solid black arrow indicates the growth plate. The open black arrow indicates growth plate cartilage bars or islands. The line black arrow indicates the metaphyseal bone. The green arrow indicates the marrow space. Blue arrows indicate red blood cells within the marrow space in the enlarged image of the junction (magnification, x20). (B) Number of marrow spaces in the untreated, PC- and PC-E-treated guinea pigs. ${ }^{*} \mathrm{P}<0.05$ vs. untreated; $\wedge \mathrm{P}<0.05$ vs. untreated and PC-E. PC, phosphocitrate; PC-E, PC- $\beta$ ethyl ester. 

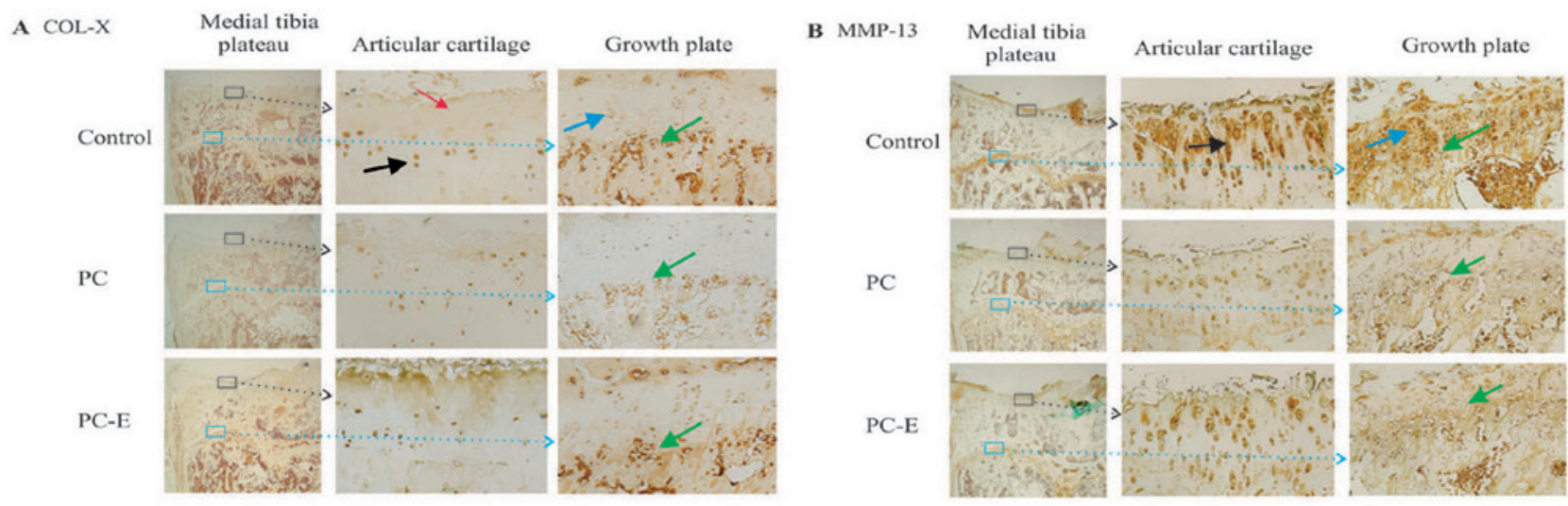

Figure 5. Levels of COL-X and MMP13 proteins (magnification, $\mathrm{x} 1$ and $\mathrm{x} 10$ ). (A) COL-X immunostaining. Areas enclosed by squares are enlarged in subsequent images. Black arrows indicate COL-X staining in the articular chondrocytes located in the deep and calcified zones in the central most degenerated area of articular cartilage. The red arrow indicates COL-X staining in the superficial and middle zone cartilaginous matrices. Blue arrows indicate COL-X staining in chondrocytes located in the maturation and hypertrophic zones. Green arrows indicate COL-X staining in cells located at the junction between the growth plate and metaphyseal bone. (B) MMP-13 immunostaining. Areas enclosed by squares are enlarged in subsequent images. The black arrow indicates MMP-13 staining in the superficial, middle and deep zones in the central most degenerated area of articular cartilage. The blue arrow indicates the growth plate. Green arrows indicate MMP-13 staining in the hypertrophic zone of growth plate. COL-X, type X collagen; MMP-13, matrix metollproteinase-13; PC, phosphocitrate; PC-E, PC- $\beta$ ethyl ester.

A COL-X

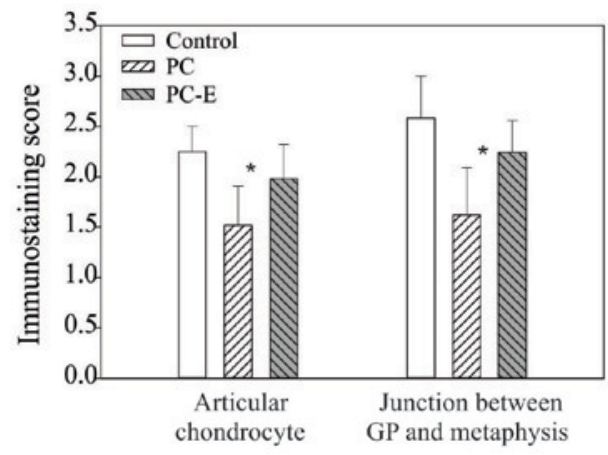

B MMP13

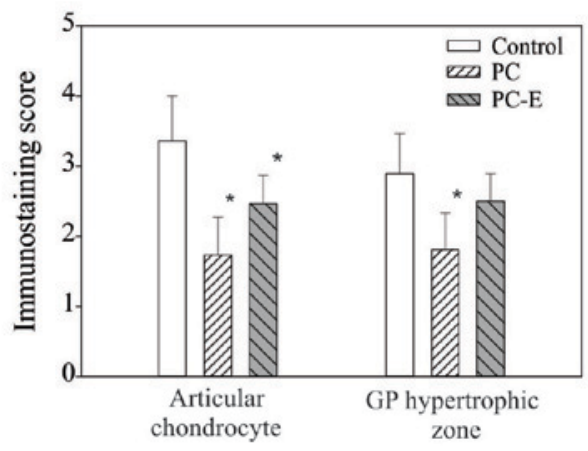

Figure 6. Immunostaining scores of the levels of COL-X and MMP13 proteins. (A) Scores for COL-X in articular chondrocytes in the control, PC- and PC-E-treated guinea pigs were $2.25 \pm 0.26,1.52 \pm 0.39$ and $1.98 \pm 0.34$, respectively. Scores for COL-X protein in the cells located at the junction between the growth plate and metaphyseal bone in the control, PC- and PC-E-treated guinea pigs were $2.58 \pm 0.42,1.62 \pm 0.49$ and $2.24 \pm 0.32$, respectively. (B) Scores for MMP-13 protein in the articular chondrocytes in the control, PC- and PC-E-treated guinea pigs were $3.36 \pm 0.64,1.73 \pm 0.55$ and $2.46 \pm 0.41$, respectively. Scores for MMP-13 protein in the cells located in hypertrophic zone of the growth plate in the untreated, PC- and PC-E-treated guinea pigs were $2.89 \pm 0.56,1.81 \pm 0.52$ and $2.50 \pm 0.39$, respectively. ${ }^{*} \mathrm{P}<0.05$ vs. control. COL-X, type X collagen; MMP-13, matrix metollproteinase-13; PC, phosphocitrate; PC-E, PC- $\beta$ ethyl ester.

score of MMP-13 protein in the articular chondrocyte was significant.

\section{Discussion}

The primary function of the calcified zone is to anchor articular cartilage to the subchondral bone (44). The current study demonstrated that severe proteoglycan loss occurred in the superficial and middle zones, as well as in the calcified zone. In addition, there were fewer articular cartilage bars and islands embedded within the subchondral bone in the untreated guinea pigs compared with the PC- or PC-E-treated guinea pigs. These results indicated that articular cartilage degeneration progressed from the articular surface and calcified zone toward the inner zones in Hartley guinea pigs. Degenerative insults to articular cartilage came from the articular side and epiphyseal side. It is conceivable that degenerative insults from the epiphyseal side may not only result in articular cartilage thinning but may also weaken the connection between the calcified cartilage and the underlying subchondral bone. It appears that the advance of calcification front and subsequent ossification or subchondral bone advance towards articular cartilage surface serves a role in articular cartilage thinning or articular cartilage destruction. This mechanism was investigated further by determining the effects of PC and PC-E on the growth plate. The results indicated that PC and PC-E inhibited growth plate thinning. The differences in growth plate thickness between the treatment groups and the control group were larger in the PC group compared with the PC-E group, suggesting that $\mathrm{PC}$ has a larger effect in inhibiting growth plate thinning, which was consistent with the inhibitory activities of PC or PC-E on articular cartilage thinning. This consistency suggests that a similar molecular mechanism may be associated with the inhibition of articular cartilage 
thinning and growth plate thinning by $\mathrm{PC}$ and its analogue PC-E.

Hypertrophic differentiation of growth plate chondrocytes and vascular invasion into the growth plate are key processes in the endochondral pathway of ossification. The current study demonstrated that high levels of two specific hypertrophic markers COL-X and MMP-13 protein, were detected in the hypertrophic zone and at the junction between the growth plate and metaphyseal bone. Their levels were decreased in the PC- or PC-E-treated guinea pigs, which indicated that $\mathrm{PC}$ and its analogue may inhibit hypertrophic differentiation of growth plate chondrocytes. In addition, there were more marrow spaces or vascular invasions at the junction between the growth plate and metaphyseal bone in the untreated guinea pigs compared with PC- or PC-E-treated guinea pigs. Consistent with their inhibitory activities on vascular invasion, PC had a greater effect on the inhibition of growth plate thinning compared with PC-E. Taken together, these results indicate that PC and its analogue PC-E inhibit endochondral pathway of ossification.

COL-X and MMP-13 protein were also detected in articular cartilage. The highest levels of COL-X were present in the articular chondrocytes located in the deep and calcified zones. These results, together with the findings that there were fewer articular cartilage bars or islands embedded within the subchondral bone in the untreated guinea pigs compared with the PC- or PC-E-treated guinea pigs, indicated that a molecular program similar to the endochondral pathway of ossification occurred in the articular cartilage. This molecular program is responsible for the degenerative insults to the articular cartilage from the epiphyseal side in the control guinea pigs. COL-X was also detected in the superficial and middle zone matrices, which suggested that this molecular program is also associated with the degenerative insults to the articular cartilage from the articular side. Therefore, it may be deduced that PC and its analogue inhibited articular cartilage thinning or destruction, in part, by inhibiting the re-activated molecular program in the OA-prone Hartley guinea pigs.

The highest levels of MMP-13 protein were detected in the superficial, middle and deep zones of articular cartilage. OA synovial fluid contains high levels of MMP-13 protein (45-47) and may be one of the sources for the superficial zone MMP-13 protein through diffusion. The strong inhibitory effect of PC on the levels of MMP-13 protein in the superficial and middle zones may explain why PC had little effect on the levels of COL-X within the extracellular matrices in these zones. Decreased MMP-13 activity in these zones due to PC treatment may result in reduced COL-X degradation (13) and therefore, preserved COL-X content in these zones.

The larger volume of growth plate cartilage in the PC-treated guinea pigs compared with the control guinea pigs suggested that PC inhibited the conversion of growth plate cartilage into bone. It was expected that there would be fewer bone matrices in the PC-treated guinea pigs compared with the untreated guinea pigs. However, this was not what was observed. A possible explanation for this contradiction is that PC may have a strong stimulatory activity on the production of extracellular matrices by chondrocytes $(27,28)$ and osteoblasts. If the stimulatory activity of PC is a dominant activity over its inhibitory activity on the conversion of growth plate cartilage into bone, formation of a larger cartilaginous template without bone loss in the PC-treated guinea pigs compared with untreated control guinea pigs would be expected.

The results presented in the present study provide further support for the hypothesis that PC or its analogue are potentially structural disease-modifying drugs for OA therapy. However, the use of PC as an orally administered OA disease modifying drug may be limited, as the small molecule PC contains a $\mathrm{P}-\mathrm{O}-\mathrm{C}$ bond and five negative charges. The $\mathrm{P}-\mathrm{O}-\mathrm{C}$ bond is vulnerable to degradation by alkaline phosphatases in vivo and the five negative charges may make PC less permeable to the intestinal membrane resulting in lower bioavailability in vivo (48). In order to overcome these limitations, two strategies are being investigated. The first strategy is to develop PC analogues that are resistant to degradation by alkaline phosphatases and/or have fewer negative changes, which will be tested as orally administered drugs on Hartley guinea pigs. The second strategy is to develop a hydrogel that controls the release or extends the release of the small molecule drug PC. One-time knee injections of the hydrogel for OA therapy will then be tested in Hartley guinea pigs prior to consideration for human clinical trials.

In conclusion, articular chondrocytes in the OA-prone Hartley guinea pigs exhibit a hypertrophic phenotype and may recapitulate a developmental molecular program similar to the endochondral pathway of ossification. This molecular program may serve a role in articular cartilage thinning and destruction. The present results indicated that PC or its analogue exerted OA disease-modifying activity, in part, by inhibiting this reactivated molecular program in OA knee joints.

\section{Reference}

1. Brown TD, Johnston RC, Saltzman CL, Marsh JL and Buckwalter JA: Posttraumatic osteoarthritis: A first estimate of incidence, prevalence, and burden of disease. J Orthop Trauma 20: 739-744, 2006.

2. Olney RC: Mechanisms of impaired growth: Effect of steroids on bone and cartilage. Horm Res 72 (Suppl 1): S30-S35, 2009.

3. Goldring MB, Otero M, Plumb DA, Dragomir C, Favero M, El Hachem K, Hashimoto K, Roach HI, Olivotto E, Borzì RM and Marcu KB: Roles of inflammatory and anabolic cytokines in cartilage metabolism: Signals and multiple effectors converge upon MMP-13 regulation in osteoarthritis. Eur Cell Mater 21: 202-220, 2011.

4. Martel-Pelletier J, Welsch DJ and Pelletier JP: Metalloproteases and inhibitors in arthritic diseases. Best Pract Res Clin Rheumatol 15: 805-829, 2001.

5. Burrage PS and Brinckerhoff CE: Molecular targets in osteoarthritis: Metalloproteinases and their inhibitors. Curr Drug Targets 8: 293-303, 2007.

6. Lotz MK, Otsuki S, Grogan SP, Sah R, Terkeltaub R and D'Lima D: Cartilage cell clusters. Arthritis Rheum 62: 2206-2218, 2010.

7. van der Kraan PM and van den Berg WB: Chondrocyte hypertrophy and osteoarthritis: Role in initiation and progression of cartilage degeneration? Osteoarthritis Cartilage 20: 223-232, 2012.

8. Tchetina EV, Squires G and Poole AR: Increased type II collagen degradation and very early focal cartilage degeneration is associated with upregulation of chondrocyte differentiation related genes in early human articular cartilage lesions. J Rheumatol 32: 876-886, 2005.

9. Bertrand J, Cromme C, Umlauf D, Frank S and Pap T: Molecular mechanisms of cartilage remodelling in osteoarthritis. Int $\mathbf{J}$ Biochem Cell Biol 42: 1594-1601, 2010.

10. Clark JM: The structure of vascular channels in the subchondral plate. J Anat 171: 105-115, 1990. 
11. Kawaguchi H: Endochondral ossification signals in cartilage degradation during osteoarthritis progression in experimental mouse models. Mol Cells 25: 1-6, 2008.

12. D'Angelo M, Yan Z, Nooreyazdan M, Pacifici M, Sarment DS, Billings PC and Leboy PS: MMP-13 is induced during chondrocyte hypertrophy. J Cell Biochem 77: 678-693, 2000.

13. Inada M, Wang Y, Byrne MH, Rahman MU, Miyaura C, López-Otín C and Krane SM: Critical roles for collagenase-3 (Mmp13) in development of growth plate cartilage and in endochondral ossification. Proc Natl Acad Sci USA 101: 17192-17197, 2004.

14. Wei F, Zhou J, Wei X, Zhang J, Fleming BC, Terek R, Pei M, Chen Q, Liu T and Wei L: Activation of Indian hedgehog promotes chondrocyte hypertrophy and upregulation of MMP-13 in human osteoarthritic cartilage. Osteoarthritis Cartilage 20: 755-763, 2012.

15. Lane LB and Bullough PG: Age-related changes in the thickness of the calcified zone and the number of tidemarks in adult human articular cartilage. J Bone Joint Surg Br 62: 372-375, 1980.

16. Oettmeier R, Abendroth K and Oettmeier S: Analyses of the tidemark on human femoral heads. II. Tidemark changes in osteoarthrosis-a histological and histomorphometric study in non-decalcified preparations. Acta Morphol Hung 37: 169-180, 1989.

17. Staines KA, Pollard AS, McGonnell IM, Farquharson C and Pitsillides AA: Cartilage to bone transitions in health and disease. J Endocrinol 219: R1-R12, 2013.

18. Williams G and Sallis JD: Structural factors influencing the ability of compounds to inhibit hydroxyapatite formation. Calcif Tissue Int 34: 169-177, 1982.

19. Shankar R, Crowden S and Sallis JD: Phosphocitrate and its analogue $\mathrm{N}$-sulpho-2-amino tricarballylate inhibit aortic calcification. Atherosclerosis 52: 191-198, 1984.

20. Sallis JD, Shankar R, Rees B and Thomson R: Protection of crystal-induced polymorphonuclear leukocyte membranolysis by phosphocitrate. Biochem Med Metab Biol 41: 56-63, 1989.

21. NairD,Misra RP, Sallis JD and Cheung HS: Phosphocitrate inhibits a basic calcium phosphate and calcium pyrophosphate dihydrate crystal-induced mitogen-activated protein kinase cascade signal transduction pathway. J Biol Chem 272: 18920-18925, 1997.

22. Cheung HS, Sallis JD and Struve JA: Specific inhibition of basic calcium phosphate and calcium pyrophosphate crystal-induction of metalloproteinase synthesis by phosphocitrate. Biochim Biophys Acta 1315: 105-111, 1996.

23. Sun Y, Reuben P, Wenger L, Sallis JD, Demadis KD and Cheung HS: Inhibition of calcium phosphate-DNA coprecipitates induced cell death by phosphocitrates. Front Biosci 10: 803-808, 2005

24. Cheung HS: Phosphocitrate as a potential therapeutic strategy for crystal deposition disease. Curr Rheumatol Rep 3: 24-28, 2001.

25. Cheung HS, Sallis JD, Demadis KD and Wierzbicki A Phosphocitrate blocks calcification-induced articular joint degeneration in a guinea pig model. Arthritis Rheum 54: 2452-2461, 2006.

26. Sun Y, Mauerhan DR, Franklin AM, Norton J, Hanley EN Jr and Gruber HE: Phosphocitrate is potentially a disease-modifying drug for noncrystal-associated osteoarthritis. Biomed Res Int 2013: 326267, 2013.

27. Sun Y, Roberts A, Mauerhan DR, Sun AR, Norton HJ and Hanley EN Jr: Biological activities of phosphocitrate: A potential meniscal protective agent. Biomed Res Int 2013: 726581, 2013.

28. Sun Y, Mauerhan DR, Steuerwald NM, Ingram J, Kneisl JS and Hanley EN Jr: Expression of phosphocitrate-targeted genes in osteoarthritis menisci. Biomed Res Int 2014: 210469, 2014.

29. Turhanen PA, Demadis KD, Peräniemi S and Vepsäläinen JJ: A novel strategy for the preparation of naturally occuring phosphocitrate and its partially esterified derivatives. J Org Chem 72 : 1468-1471, 2007

30. Bendele AM, White SL and Hulman JF: Osteoarthrosis in guinea pigs: Histopathologic and scanning electron microscopic features. Lab Anim Sci 39: 115-121, 1989.

31. Kuyinu EL, Narayanan G, Nair LS and Laurencin CT: Animal models of osteoarthritis: Classification, update and measurement of outcomes. J Orthop Surg Res 11: 19, 2016.

32. Poole R, Blake S, Buschmann M, Goldring S, Laverty S, Lockwood S, Matyas J, McDougall J, Pritzker K, Rudolphi K, et al: Recommendations for the use of preclinical models in the study and treatment of osteoarthritis. Osteoarthritis Cartilage 18 (Suppl 3): S10-S16, 2010.
33. Jimenez PA, Glasson SS, Trubetskoy OV and Haimes HB: Spontaneous osteoarthritis in Dunkin Hartley guinea pigs: Histologic, radiologic and biochemical changes. Lab Anim Sci 47: 598-601, 1997

34. Huebner JL, Seifer DR and Kraus VB: A longitudinal analysis of serum cytokines in the Hartley guinea pig model of osteoarthritis. Osteoarthritis Cartilage 15: 354-356, 2007.

35. Thomsen JS, Straarup TS, Danielsen CC, Oxlund H and Brüel A: No effect of risedronate on articular cartilage damage in the Dunkin Hartley guinea pig model of osteoarthritis. Scand J Rheumatol 42: 408-416, 2013.

36. Thomsen JS, Straarup TS, Danielsen CC, Oxlund H and Brüel A: Relationship between articular cartilage damage and subchondral bone properties and meniscal ossification in the Dunkin Hartley guinea pig model of osteoarthritis. Scand J Rheumatol 40 : 391-399, 2011.

37. Horcajada MN, Sanchez C, Membrez Scalfo F, Drion P, Comblain F, Taralla S, Donneau AF, Offord EA and Henrotin Y: Oleuropein or rutin consumption decreases the spontaneous development of osteoarthritis in the Hartley guinea pig. Osteoarthritis Cartilage 23: 94-102, 2015.

38. Sun Y, Haines N, Roberts A, Ruffolo M, Mauerhan DR, Mihalko KL, Ingram J, Cox $M$ and Hanley EN Jr: Disease-modifying effects of phosphocitrate and phosphocitrate-beta-ethyl ester on partial meniscectomy-induced osteoarthritis. BMC Musculoskelet Disord 16: 270, 2015.

39. Huebner JL, Hanes MA, Beekman B, TeKoppele JM and Kraus VB: A comparative analysis of bone and cartilage metabolism in two strains of guinea-pig with varying degrees of naturally occurring osteoarthritis. Osteoarthritis Cartilage 10: 758-767, 2002.

40. Sun Y, Mauerhan DR, Kneisl JS, Norton HJ, Zinchenko N and Ingram JA: Histologic evidence for collagen and proteoglycan changes in menisci of osteoarthritic patients. In: Proceedings of the ACR/ARHP 75th Annual Scientific Meeting, Chicago, IL, 2011.

41. Kirsch T, Swoboda B and Nah H: Activation of annexin II and $\mathrm{V}$ expression, terminal differentiation, mineralization and apoptosis in human osteoarthritic cartilage. Osteoarthritis Cartilage 8: 294-302, 2000

42. Kim HA, Lee YJ, Seong SC, Choe KW and Song YW: Apoptotic chondrocyte death in human osteoarthritis. J Rheumatol 27: 455-462, 2000.

43. Kouri JB, Aguilera JM, Reyes J, Lozoya KA and González S: Apoptotic chondrocytes from osteoarthrotic human articular cartilage and abnormal calcification of subchondral bone. J Rheumatol 27: 1005-1019, 2000.

44. Sophia Fox AJ, Bedi A and Rodeo SA: The basic science of articular cartilage: Structure, composition and function. Sports Health 1: 461-468, 2009.

45. Marini S, Fasciglione GF, Monteleone G, Maiotti M, Tarantino U and Coletta M: A correlation between knee cartilage degradation observed by arthroscopy and synovial proteinases activities. Clin Biochem 36: 295-304, 2003.

46. Ozler K, Aktas E, Atay C, Yilmaz B, Arikan M and Güngör S: Serum and knee synovial fluid matrix metalloproteinase-13 and tumor necrosis factor-alpha levels in patients with late-stage osteoarthritis. Acta Orthop Traumatol Turc 50: 356-361, 2016.

47. Molloy ES, Morgan MP, McDonnell B, O'byrne J and McCarthy GM: BCP crystals increase prostacyclin production and upregulate the prostacyclin receptor in OA synovial fibroblasts: Potential effects on mPGES1 and MMP-13. Osteoarthritis Cartilage 15: 414-420, 2007.

48. Cooper CM and Sallis JD: Studies on the gastrointestinal absorption of phosphocitrate, a powerful controller of hydroxyapatite formation. Int J Pharm 98: 165-172, 1993.

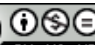

This work is licensed under a Creative Commons Attribution-NonCommercial-NoDerivatives 4.0 International (CC BY-NC-ND 4.0) License. 